\title{
Parasternal intercostal electromyography: a novel tool to assess respiratory load in children
}

\author{
Victoria MacBean', Caroline J. Jolley' ${ }^{1}$ Timothy G. Sutton', Akash Deep², Anne Greenough ${ }^{1,3}$, John Moxham and \\ Gerrard F. Rafferty'
}

BACKGROUND: Parasternal intercostal muscle electromyography (EMGpara) represents a novel tool to assess respiratory load when volitional techniques are not possible. This study examined the application of EMGpara in healthy, wheezy, and critically ill children.

METHODS: Surface EMGpara was measured during tidal breathing in 92 healthy children, 20 wheezy preschool children (with measurements repeated following bronchodilator), and 25 mechanically ventilated children during supported ventilation and on continuous positive airways pressure.

RESULTS: EMGpara was related to age, height, and weight in the healthy group ( $r=-0.623,-0.625,-0.641$ respectively, all $P<0.0001$ ). An age-based equation for predicted EMGpara was developed and patient data expressed as z-scores. EMGpara was higher in wheezy children prebronchodilator than healthy controls (median interquartile range (IQR) z-score 0.53 (0.07-1.94), $P=0.0073)$, falling to levels not different to healthy children postbronchodilator $(-0.08(-0.50-1.00))$. In the critically ill children, EMGpara was higher $(P<0.0001)$ than in healthy subjects during both mechanical ventilation (median (IQR) z-score 1.14 (0.331.93)) and continuous positive airways pressure (1.88 (0.91-3.03)). CONCLUSION: EMGpara is feasible in children and infants both healthy and diseased, is raised in those with elevated respiratory load, and is responsive to clinical interventions. EMGpara represents a potential method to assess respiratory status in patients conventionally challenging to assess.

A ccurate assessment of respiratory function is crucial for optimal disease management in a range of settings, and volitional testing methods are frequently used. Certain patient populations such as young children and those receiving intensive care are, however, unable to comply with such testing. Assessment of such patient groups therefore revolves primarily around clinical examination and basic physiological parameters. Clinical examination, while essential in the evaluation of any patient, often relates poorly to objective assessment of disease state and clinical outcome (1-3) and should therefore be supplemented with tools to objectively quantify the severity of respiratory disease. The development of effort-independent markers of respiratory function is therefore of substantial importance (4).

A number of nonvolitional techniques have been developed to assess various aspects of respiratory function, such as the forced oscillation technique, plethysmographic airways resistance, lung clearance index and respiratory interrupter resistance (4). Although feasible in older children and adults, these techniques still require passive cooperation from the subject in order to maintain steady tidal breathing with a leak-free seal around the mouthpiece or mask. Successful measurements may be challenging in younger children, and may require sedation for use in infants. Many of these techniques are also unsuitable for use in critically ill populations.

Ventilation is dependent on the load placed on the respiratory system, the force generating capacity of the respiratory muscles, and the neural output from brainstem respiratory centers (neural respiratory drive (NRD)). NRD increases in parallel with respiratory load in order to maintain adequate levels of ventilation for blood gas homeostasis (5). NRD can be recorded from the electromyogram (EMG) of selected important respiratory muscles. While diaphragm EMG provides a sensitive measure of NRD which reflects the load on the respiratory muscles (6), obtaining uncontaminated signals requires the passage of an esophageal catheter, making the technique unsuited to routine clinical use, particularly in pediatrics. The parasternal intercostal muscles are obligate inspiratory muscles, recruited in tandem with the diaphragm (7), whose anatomical location results in them being well suited to the measurement of NRD. Parasternal intercostal muscle electromyography (EMGpara) therefore provides a noninvasive measure of NRD that potentially could be used to assess lung disease severity and guide patient management in children with a range of conditions. We have previously demonstrated that EMGpara is responsive to changes in respiratory load in healthy adult subjects (8) and provides a noninvasive marker of neural drive that reflects disease severity and exercise-induced breathlessness in adult patients with cystic fibrosis (9).

'Department of Respiratory Medicine, Division of Asthma Allergy and Lung Biology, King's College London, London, UK; ${ }^{2}$ Paediatric Intensive Care Unit, King's College Hospital NHS Foundation Trust, London, UK; ${ }^{3}$ National Institute for Health Research (NIHR) Biomedical Research Centre based at Guy's and St Thomas' NHS Foundation Trust and King's College London, London, UK. Correspondence: Victoria MacBean (victoria.macbean@kcl.ac.uk)

Received 12 November 2015; accepted 22 February 2016; advance online publication 11 May 2016. doi:10.1038/pr.2016.89 


\section{Articles | MacBean etal.}

The aims of this study were, therefore, to examine the feasibility of measuring EMGpara in a range of pediatric populations, to explore the factors influencing EMGpara in healthy children of a range of ages, to compare EMGpara between healthy children and those with respiratory disease, and to examine the EMGpara response to increases and decreases in respiratory load.

Some of these data have previously been shown in abstract form, in the context of interim analyses of each cohort (10-12).

\section{RESULTS}

In total, 92 healthy children, 20 children with preschool wheeze and 25 children receiving intensive care were studied. Subject characteristics are presented in Table 1, with additional data for the Pediatric Intensive Care Unit (PICU) cohort presented in Table 2. Example EMGpara traces before and after bronchodilator administration are shown in Figure 1.

\section{Healthy Children}

Median interquartile range (IQR) EMGpara in the cohort of 92 healthy children was $4.91(3.67-6.59) \mu \mathrm{V}$. There were more female $(n=57)$ than male $(n=35)$ subjects within the healthy cohort, although height, weight, age, and BMI were not significantly different between sexes. There was no significant difference in median (IQR) EMGpara between male and female healthy children (5.61 (3.64-8.48) $\mu \mathrm{V}$ vs. $4.76(3.70-6.26) \mu \mathrm{V}, P=0.189)$.

Of the 92 healthy children, 56 were able to reliably perform maximal inspiratory efforts to TLC. Median (IQR) EMGpara\%max for this cohort of 56 children was 6.80 (5.06$10.16) \%$. The corresponding median (IQR) EMGpara for the same 56 children was $4.46(3.56-6.04) \mu \mathrm{V}$. The median (IQR) age of those children who could reliably perform such maneuvers was significantly higher than those who could not (11.07 (7.85-14.16) vs. $5.37(2.65-13.53)$ years, $P=0.0112)$.

Table 1. Subject characteristics for healthy, wheezy, and PICU groups

\begin{tabular}{|c|c|c|c|}
\hline & $\begin{array}{l}\text { Healthy subjects } \\
\qquad(n=92)\end{array}$ & $\begin{array}{l}\text { Preschool wheeze } \\
\text { patients }(n=20)\end{array}$ & $\begin{array}{c}\text { PICU patients } \\
(n=25)\end{array}$ \\
\hline Age (y) & $9.51(5.14-13.99)$ & $3.70(2.23-4.43)$ & $1.00(0.40-5.80)$ \\
\hline Sex (M:F) & $35: 57$ & $12: 8$ & $12: 13$ \\
\hline Weight (kg) & $32.40(20.79-54.25)$ & $15.03(13.46-16.40)$ & $9.3(5.25-26.00)$ \\
\hline Height (m) & $1.43(1.13-1.64)$ & $0.98(0.91-1.01)$ & $\begin{array}{l}\text { Data not } \\
\text { available }\end{array}$ \\
\hline $\mathrm{BMI}\left(\mathrm{kg} / \mathrm{m}^{2}\right)$ & $17.05(15.84-21.54)$ & $15.94(15.28-17.03)$ & $\begin{array}{l}\text { Data not } \\
\text { available }\end{array}$ \\
\hline $\begin{array}{l}\text { BMI-for-age } \\
\text { percentile }\end{array}$ & $64.4(40.2-85.7)$ & $51.5(22.2-86.3)$ & $\begin{array}{l}\text { Data not } \\
\text { available }\end{array}$ \\
\hline \multirow{4}{*}{$\begin{array}{l}\text { Ethnicity } \\
(n(\%))\end{array}$} & White: 58 (63.0) & White: 13 (65.0) & White: 14 (56.0) \\
\hline & Black: 22 (23.9) & & \\
\hline & Asian: 6 (6.5) & Black: 4 (20.0) & Black: 3 (12.0) \\
\hline & Other: 6 (6.5) & Other: 3 (15.0) & Asian: 8 (32.0) \\
\hline
\end{tabular}

Data are shown as median (IQR).

PICU, Pediatric Intensive Care Unit.
In the cohort as a whole, significant negative correlations were found between EMGpara and age $(r=-0.623)$, weight $(r=-0.641)$, and height $(r=-0.625$, all $P<0.0001$, Figure 2$)$. Excluding those children who were unable to perform maximal inspiratory efforts led to a small reduction in the strength of these relationships but they remained highly significant $(r=-0.530,-0.599$, and -0.555 respectively, all $P<0.0001)$. In contrast, no significant relationships were observed between EMGpara\%max and age, weight or height (all $P>0.2$ ). No significant relationships were seen between EMGpara recorded during the maximal inspiratory maneuver and age or height, although there was a weak correlation between maxEMGpara and weight $(r=-0.332, P=0.013)$. Application of the WaldWolfowitz runs test indicated that there was no significant deviation from linearity in any of the above relationships, and that the data were therefore best suited to linear rather than curvilinear models.

As nonnormal distribution was observed in both the EMGpara data and the residuals plot, logarithmic transformation of the EMGpara values was performed to allow the assumptions of linear regression to be met. Age, height, and weight were all strongly colinear and therefore, were not considered suitable as independent predictors of logEMGpara. Age demonstrated the best regression model fit $\left(R^{2}=0.376\right.$, compared with $R^{2}=0.348$ for logEMGpara vs. height and $R^{2}=0.332$ for logEMGpara vs. weight respectively), therefore predicted logEMGpara was derived from age using the equation:

Predicted logEMGpara $=-0.02347 \times$ age +0.9332

The $95 \%$ confidence intervals for the regression coefficient were -0.0298 to -0.0171 , and for the $y$-intercept 0.866 to 1.001 .

\section{Comparison to Patient Populations and Response to Change} Wheezy preschool children. Median (IQR) EMGpara at baseline (prior to bronchodilator administration) in the 20 children with preschool wheeze was $8.20(7.01-13.17) \mu \mathrm{V}$. This corresponded to a median (IQR) logEMGpara z-score of 0.53 (0.07-1.94). Of the 20 children, 6 (30\%) demonstrated abnormally elevated EMGpara, defined as a logEMGpara $>1.645$ $\mathrm{z}$-scores above the predicted logEMGpara derived from the

Table 2. Clinical characteristics of 25 PICU children

\begin{tabular}{lc}
\hline Peak inspiratory pressure $\left(\mathrm{cmH}_{2} \mathrm{O}\right)$ & $14(12-15)$ \\
Positive end expiratory pressure $\left(\mathrm{cmH}_{2} \mathrm{O}\right)$ & $5(5-6)$ \\
Duration of mechanical ventilation $(\mathrm{d})$ & $5(2-7)$ \\
Fraction of inspired oxygen & $0.30(0.26-0.35)$ \\
Primary reason for admission & Respiratory failure $(n=7)$ \\
& Liver transplant $(n=8)$ \\
& Other abdominal surgery $(n=4)$ \\
& Neurological $/$ neurosurgical \\
condition $(n=5)$ \\
Abdominal sepsis $(n=1)$
\end{tabular}

Data are shown as median (IQR).

PICU, Pediatric Intensive Care Unit. 
a

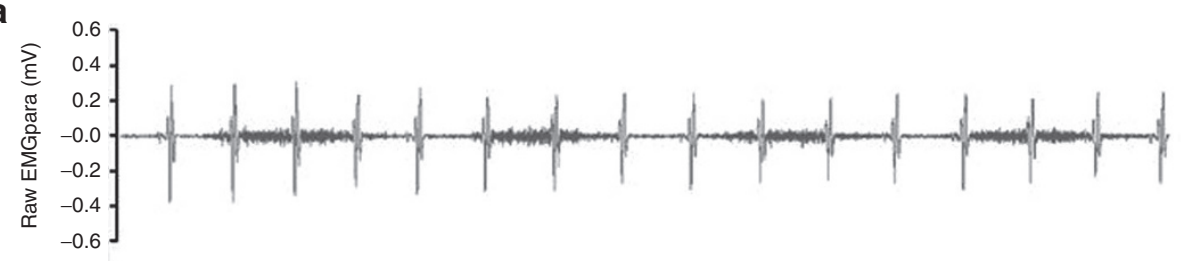

b

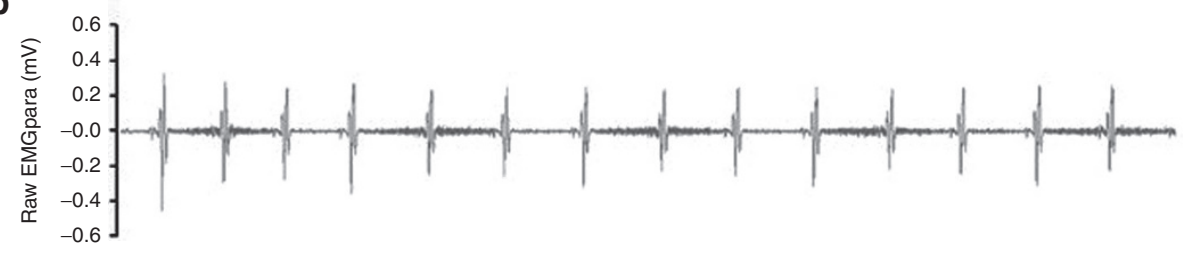

Figure 1. Representative traces of EMGpara before (a) and after (b) bronchodilator administration.
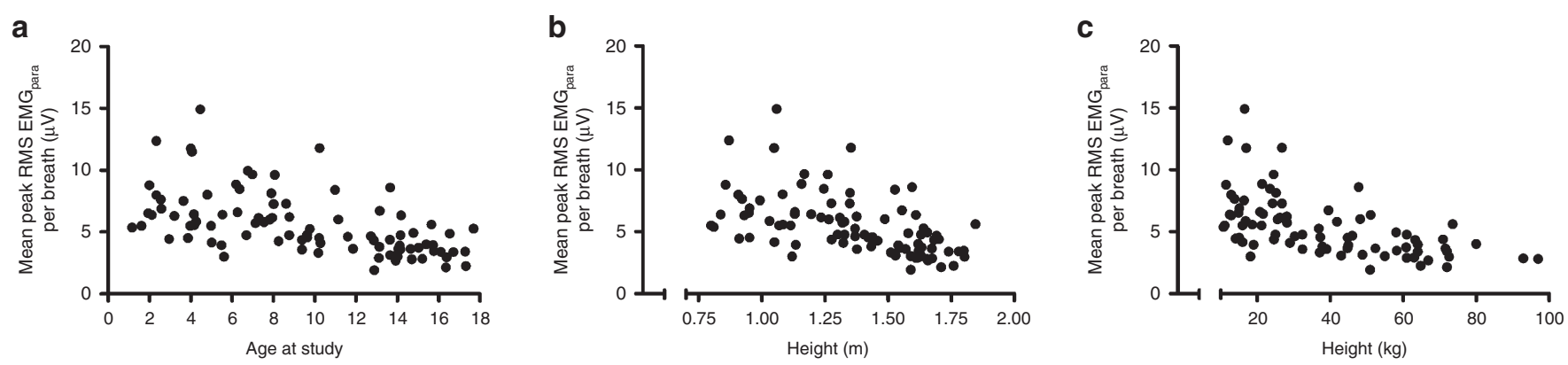

Figure 2. Relationships between EMGpara and age (a), height (b), and weight (c) in 92 healthy children.

healthy cohort. None of the wheezy children could reliably perform maximal inspiratory maneuvers.

Median (IQR) EMGpara fell significantly following administration of salbutamol to $6.98(6.01-10.48) \mu \mathrm{V}(P=0.0037)$. The postbronchodilator value corresponded to a median (IQR) logEMGpara z-score of $-0.08(-0.50-1.00)$. Only three children (15\%) demonstrated elevated EMGpara post-salbutamol, all of whom had elevated logEMGpara at baseline. logEMGpara was higher in wheezy children than predicted at baseline $(P=0.0073)$, but not post-bronchodilator.

The within-subject interminute coefficient of variation was $13.4 \%$ in wheezy children and $11.2 \%$ in the healthy cohort. Multiplying the CV from the wheezy children by 1.65 gave a threshold for significant bronchodilator (BD) response of $22.1 \%$. Using this threshold, seven of the 20 preschool wheezy children (35\%) showed a significant reduction in EMGpara following bronchodilator administration.

Mechanically ventilated PICU patients. Comparisons were made to levels of EMGpara observed during minimally supported breathing while on continuous positive airways pressure (CPAP) in the PICU children, as this condition best represented the respiratory system under load. Median (IQR) EMGpara on CPAP was 12.7 (9.9-17.9) $\mu \mathrm{V}$ which corresponded to a median (IQR) logEMGpara z-score of 1.88 $(0.91-3.03)$. Fourteen $(56 \%)$ of the children had an elevated logEMGpara, >1.645 z-scores above the predicted value from healthy children. A weak inverse relationship was observed between age and EMGpara measured both during mechanical ventilation $(\mathrm{MV})(r=-0.560, P=0.004)$ and CPAP $(r=$ $-0.402, P=0.046)$, reflecting the findings from the healthy cohort. When receiving MV median (IQR) logEMGpara was 1.14 (0.33-1.93), with nine children (36\%) demonstrating abnormally elevated EMGpara. logEMGpara was significantly higher than predicted under both conditions $(P<0.0001)$.

Both EMGpara and neuroventilatory efficiency (NVE) were significantly different on MV compared with CPAP (Table 3). No significant changes in respiratory rate (RR), tidal volume $\left(V_{T}\right)$ or respiratory duty cycle (Ti:Ttot) were observed when patients were switched from MV to CPAP (Table 3). Neither EMGpara nor NVE when measured at baseline on MV or change in either variable when switched to CPAP were related to duration of MV (all $r<0.26, P>0.2$ ).

Three children failed extubation and were subsequently reintubated. Increased work of breathing with inability to maintain blood oxygenation was documented as the reason for reintubation in all three cases, with one child additionally experiencing pulmonary edema and elevated $\mathrm{CO}_{2}$ levels. All three demonstrated elevated EMGpara both during MV and while on CPAP. Thresholds for predicting extubation outcome, together with sensitivity and specificity values and area under the receiver operator characteristic curve for each measured variable are shown in Table 4. NVE during CPAP was the strongest predictor of extubation outcome, using a threshold 
Articles | MacBean et al.

Table 3. Comparison of respiratory variables during MV and spontaneous breathing on CPAP in 25 PICU patients

\begin{tabular}{lcccc}
\hline & MV & CPAP & Pvalue & Change from MV to CPAP (\%) \\
\hline EMGpara $(\mu \mathrm{V})$ & $10.4(7.4-14.5)$ & $12.7(9.9-17.9)$ & 0.0001 & $16.7(2.1-34.0)$ \\
NVE $(\mathrm{ml} / \mathrm{kg} / \mu \mathrm{V})$ & $0.77(0.46-1.11)$ & $0.57(0.40-0.79)$ & 0.0001 & $-18.4(-32.0-0.1)$ \\
RR & $35(24-48)$ & $35(27-47)$ & 0.178 & $2.7(-5.2-18.6)$ \\
Tidal volume $(\mathrm{ml} / \mathrm{kg})$ & $7.0(6.6-9.5)$ & $7.5(6.2-9.7)$ & 0.628 & $-2.0(-11.9-11.5)$ \\
Ti:Ttot & $0.37(0.34-0.41)$ & $0.39(0.35-0.45)$ & 0.279 & $3.3(-3.5-10.0)$ \\
\hline
\end{tabular}

Data are presented as median (IQR).

CPAP, continuous positive airways pressure; EMGpara, parasternal intercostal electromyogram; MV, mechanical ventilation; NVE, neuroventilatory efficiency; RR, respiratory rate; Ti,Ttot: respiratory duty cycle.

Table 4. Maximum sensitivity and specificity of individual variables in predicting extubation failure, with areas under the receiver operating characteristics curve for 25 PICU patients

\begin{tabular}{lccc}
\hline Parameter & Threshold & $\begin{array}{c}\text { Sensitivity/ } \\
\text { specificity }\end{array}$ & $\begin{array}{c}\text { Area under } \\
\text { ROC curve }\end{array}$ \\
\hline NVE (CPAP) & $<0.43 \mathrm{ml} / \mathrm{kg} / \mu \mathrm{V}$ & $100 / 81.8$ & 0.947 \\
NVE (MV) & $<0.57 \mathrm{ml} / \mathrm{kg} / \mu \mathrm{V}$ & $77.3 / 100$ & 0.917 \\
EMGpara (CPAP) & $>14.8 \mu \mathrm{V}$ & $100 / 72.7$ & 0.879 \\
EMGpara (MV) & $>14.0 \mu \mathrm{V}$ & $81.8 / 100$ & 0.879 \\
$V_{\mathrm{T}}$ (CPAP) & $<6.4 \mathrm{ml} / \mathrm{kg}$ & $100.72 / 7$ & 0.742 \\
$\Delta$ NVE (MV to CPAP) & $>-18.4 \%$ & $100 / 59.1$ & 0.697 \\
RSBI (MV) & $>3.84$ & $100 / 50.0$ & 0.697 \\
Ti:Ttot (CPAP) & $>0.34$ & $86.4 / 66.7$ & 0.682 \\
RSBI (CPAP) & $>4.34$ & $100 / 54.6$ & 0.682 \\
$V_{\mathrm{T}}$ (MV) & $<7.21 \mathrm{ml} / \mathrm{kg}$ & $100 / 54.6$ & 0.667 \\
RR (CPAP) & $>40 \mathrm{bpm}$ & $66.7 / 72.7$ & 0.606 \\
Ti:Ttot (MV) & $>0.40$ & $100 / 36.4$ & 0.591 \\
RR (MV) & $>41 \mathrm{bpm}$ & $66.7 / 68.2$ & 0.576 \\
$\Delta$ EMGpara (MV to CPAP) & $<9.7 \%$ & $66.7 / 63.6$ & 0.546 \\
\hline
\end{tabular}

CPAP, continuous positive airways pressure; EMGpara, parasternal intercostal electromyogram; NVE, neuroventilatory efficiency; MV, mechanical ventilation; $\mathrm{PICU}$ Pediatric Intensive Care Unit; ROC, receiver operator characteristic; $\mathrm{RSBI}$, rapid shallow breathing index; $R R$, respiratory rate; $V_{T}$, tidal volume; Ti:Ttot, respiratory duty cycle.

of $0.43 \mathrm{ml} / \mathrm{kg} / \mu \mathrm{V}$. NVE on MV and EMGpara on MV or on CPAP also performed well, with existing indices demonstrating poorer predictive ability.

\section{DISCUSSION}

These studies have demonstrated the feasibility of measuring EMGpara in children of a range of ages, both in healthy and diseased states. The data from healthy children have shown important age-related changes in EMGpara and are sufficient for comparative purposes against the patient data acquired in the current study. EMGpara has been shown to be sensitive to change in respiratory load induced by bronchodilation in the cohort of children with wheeze and by removal of inspiratory pressure support in critically ill children. EMGpara, as a measure of NRD, therefore demonstrates potential value in the assessment of disease severity and physiological responses to treatment in pediatric respiratory and critical care, providing a valuable aid to patient management.
A commonly reported limitation of surface EMG recordings is the inherently poor between-subject and between-occasion reproducibility, resulting from differences in body composition and electrode placement $(9,13,14)$. To overcome this, previous studies have expressed resting EMGpara as a percentage of that obtained during a maximal maneuver (EMGpara\%max). Such an approach, however, limits the technique to those subjects able to reliably perform volitional efforts and renders the technique unsuitable for use in young children and infants, as well as patients who are unable to perform such maneuvers as a result of fatigue, pain, poor comprehension or motivation. In our study, the difficulties associated with acquiring reliable maximal EMGpara signals were highlighted by the finding that such measures could only be obtained in 56 of the 92 healthy subjects, and in none of the preschool children with wheeze. Similarly, achieving reliable maximal inspiratory efforts is difficult in intubated, sedated pediatric patients, and can only be obtained by using maintained endotracheal tube (ETT) occlusion (15). ETT occlusion may not, however, represent a suitable method for obtaining maximal EMGpara as inhibition of parasternal intercostal muscle activity during static (isovolume) inspiratory maneuvers has been demonstrated (16). For this reason, maxEMGpara was not recorded in our PICU cohort. Our data suggest, however, that EMGpara recorded during tidal breathing in children provides a simple and feasible technique to assess NRD without the need for complex respiratory maneuvers. Data regarding interoccasion variability of the EMGpara signal would be required to facilitate the use of EMGpara as a longitudinal monitoring tool. Although we have not investigated this in the current study, previous investigators $(17,18)$ have demonstrated the measure to be reproducible in pediatric populations.

The changes observed in EMGpara with age are similar to those previously demonstrated using occlusion pressure (19) and are likely to be due to the substantial maturational changes that occur in the respiratory system during childhood. Decreases in airways resistance as a result of lung and airway growth, together with increased alveolarisation and vascularization and ribcage ossification act to decrease the load on the respiratory system and hence lead to reductions in the NRD required to maintain adequate ventilation. That these changes are observed when using raw (non-normalized) EMGpara signals further supports the use of raw EMGpara as a marker of NRD. 
It is possible that the increase in skin thickness (and thus muscle-electrode distance) occurring with somatic growth may also contribute to the observed decreases in EMGpara with increasing age. Of note, no relationship between age and the magnitude of maxEMGpara was observed in the 56 children able to complete maximal inspiratory efforts satisfactorily, suggesting that the electrical impedance of the skin and subcutaneous tissue is unlikely to be the explanation for falling EMGpara activity with increasing age. Although data regarding the relationship between EMGpara and maturational changes in pulmonary function, skin and chest wall tissue properties would be valuable, extremely large sample sizes would be required to examine the relative contribution of these variables. Such studies will only become feasible with the development of automated algorithms for EMGpara signal processing and analysis.

Our study used an age-based prediction equation, thereby accounting for maturational changes. Many of the PICU children were younger than those included in the healthy cohort, therefore the reference equation was extrapolated to encompass the lower age range. We recognize that this approach is not entirely satisfactory, but has been widely used previously in the context of other pulmonary function tests such as spirometry. Obtaining EMGpara values from healthy infants would be important in future studies to facilitate more accurate comparison between healthy children and patient groups and to avoid the need for extrapolation of reference equations. Similarly, the effect of posture on EMGpara has not yet been investigated in children and we recognize the limitation of comparing EMGpara values obtained from healthy children in the seated position to those from critically ill children measured in the supine posture.

The importance of obtaining normative data from healthy infants is particularly important in light of the subtle differences in activation patterns between the diaphragm and the second interspace parasternal intercostal muscles known to be present in adults (20). The presence of a craniocaudal gradient in the onset of inspiratory activity in the human parasternal intercostal muscles emphasizes the need for standardization of electrode positioning, and the current study utilized the same technique as previously published work $(8,9,14,18,21-24)$. Recording electrodes were placed directly adjacent to the sternal border in the second intercostal space bilaterally, with the positive electrode on the right in all patients. Developmental changes in the relative activation of inspiratory muscles have not yet been investigated, or have the relative responses under conditions of elevated load. There is some disagreement within the literature regarding the response of the parasternal intercostal muscles to increases in respiratory load: Roussos et al. (25) demonstrated alternating contributions of the diaphragm and intercostal muscles during inspiratory loading tasks, whereas Reilly et al. showed consistent increases in both muscle groups during incremental exercise, with linear increases in parasternal intercostal activity (9). Detailed clinical and laboratory-based studies would be required to fully understand such recruitment patterns, taking into account of the ongoing anatomical and biomechanical changes occurring with growth and development.

\section{Clinical Application of EMGpara}

This study has examined the ability of EMGpara to discriminate between healthy children and those with respiratory pathology, as well as performing exploratory analysis of the predictive ability of EMGpara with respect to extubation outcome and the response of the measurement to bronchodilation.

The major advantages of EMGpara are its applicability to a wide range of clinical settings, and across the span of ages encountered in pediatric respiratory care. The children included in this study ranged from newborn to adolescent, and measurements were made without any requirement for sedation. Although some portions of recordings were contaminated by movement or speech, these were easily excluded from analysis and obtaining at least $1 \mathrm{~min}$ of uncontaminated recording was possible in all of the 137 children studied. The ability to utilize EMGpara in such a range of ages and clinical settings allows a contiguous reference range to be developed and should allow accurate clinical assessment of respiratory status from the intensive care unit to the outpatient clinic.

One clinical area in which the utility of EMGpara requires careful consideration is that of neuromuscular disease. The extent of respiratory compromise is unlikely to be reflected by the magnitude of the EMGpara signal in the presence of neuromuscular abnormalities. In such cases, it may be necessary to express the resting signal as a percentage of that obtained during a maximal maneuver and provide a measure of the load on the respiratory system relative to the capacity of the respiratory muscles. Further studies should seek to investigate the relevance of measures of neural drive in neuromuscular disease populations.

The elevated EMGpara observed in both the wheezy and PICU groups when compared with healthy controls suggests that a single measure of EMGpara may provide an indication of increased respiratory load, and may therefore have potential as a marker of disease severity. EMGpara has previously been shown to relate to disease severity, as defined by forced expiratory volume in $1 \mathrm{~s}$, in adults with cystic fibrosis (9) and to reflect variability of airflow obstruction in asthmatic adults (13). In the context of the populations included in the current study, levels of EMGpara may be able to guide changes in ventilatory support, or aid in determining the severity of wheezing illness. Earlier studies in pediatric cohorts have demonstrated EMGpara to be responsive to change in respiratory load induced by histamine challenge, with values returning to baseline following bronchodilator administration $(18,24)$. These investigators, however, expressed EMGpara values relative to the patients' own baseline values and while this is a robust method for assessing intraindividual change, it does not allow comparison to normative values. Our study therefore builds on this previous work and brings the technique closer to clinical translation.

The technique could also provide a useful objective assessment tool to assess response to $\mathrm{BD}$. The absence of objective 
pulmonary function tests has been highlighted as a barrier to optimal treatment in wheezy preschool children (26). While techniques such as respiratory interrupter resistance and forced oscillation technique have entered clinical use in some centers, the practicalities of obtaining technically acceptable data in young patient populations limit the wider uptake of such measures. EMGpara measurements could be used to support clinical judgement when assessing the benefit of $\mathrm{BD}$ administration in this population, further enhancing diagnosis and helping to guide treatment decisions. The preschool wheeze cohort in this study comprised an unselected group, with a range of disease severity and control. A substantial bronchodilator response was not to be expected in all of the subjects studied, and indeed a change in excess of the determined threshold was observed in only seven of the 20 subjects. This may reflect good disease control rather than insufficient sensitivity of the technique; indeed previous data demonstrate a range of magnitudes of bronchodilator response in asthmatic preschool children, quantified by measures of airways resistance (27). Future studies could consider examining EMGpara-based bronchodilator response in a more homogeneous group of steroid-naive preschool children, in whom disease control may be poorer.

The PICU cohort was relatively small and clinically heterogeneous, and with only three children failing extubation no firm conclusions can be drawn in terms of the utility of the technique in guiding extubation timing. This was undertaken as an exploratory analysis only and we do not suggest this to be statistically robust. Nonetheless, the fact that EMGpara-based parameters performed better in this cohort than indexes such as the rapid shallow breathing index at predicting extubation outcome suggests that EMGpara may have promise in this field. Larger studies would allow age-specific thresholds to be developed to indicate likely extubation readiness, facilitating clinical translation of this measure. Such studies would also allow subgroup analysis by clinical presentation, and may reveal differences between patients with and without a primary respiratory reason for admission.

\section{Directions for Future Research}

The promising performance of EMGpara in the current study suggests that the technique warrants continued investigation. Future studies should examine in more detail the technical and methodological factors influencing measurement of EMGpara, including the effect of posture and individual anthropometric characteristics (with particular focus on skin and adipose layer thickness). Much larger sample sizes should be recruited in order to develop robust reference ranges, encompassing the full age range from infancy upward. Future work could also include more detailed clinical and physiological characterization of patients and relating clinical assessment findings to EMGpara values, which would allow EMGpara to be more clearly contextualized with respect to the clinical setting. All of the aforementioned studies will be facilitated by development of automated analysis systems.

\section{Conclusion}

$\mathrm{NRD}$, quantified by the EMG of the parasternal intercostal muscles, is feasible in children and infants across the spectrum of health and disease. EMGpara is raised compared with healthy children in those with elevated respiratory load, and is responsive to clinical interventions. EMGpara represents a potential method to assess respiratory status in a population of children for whom currently available measures have limited application.

\section{METHODS}

The study was granted ethical approval (National Research Ethics Committees London-Dulwich and London-Fulham) and conformed to the principles of the Declaration of Helsinki. Parents or caregivers of all participants gave informed written consent.

\section{Healthy Controls}

Healthy children (under the age of 18 y) with no history of cardiorespiratory or neurological disease were recruited via friends and family of research staff, students, and from a database of previous research participants. Any children exhibiting symptoms of an acute viral respiratory tract infection (cough and/or coryza) were excluded.

\section{Wheezy Children}

Children under the age of $5 \mathrm{y}$ with a physician diagnosis of asthma or persistent wheeze were identified from the pediatric respiratory clinics at King's College Hospital. Children with any additional respiratory, cardiac or neurological diagnosis likely to impact on respiratory drive were excluded.

\section{PICU Patients}

Patients were recruited from the PICU at King's College Hospital. Children who had been mechanically ventilated for $>24 \mathrm{~h}$ were eligible for inclusion. Children extubated for palliation were excluded. Measurements were performed once the child had been deemed ready for extubation by the clinical team. Extubation failure was defined as reintubation within $48 \mathrm{~h}$ of extubation. Criteria for reintubation were as described earlier (28). Any decision to extubate or, where relevant, reintubate was made by the clinical team independent of any study measurements. Clinicians were unaware of the child's response to the spontaneous breathing trial and were blinded to all data acquired during the course of the study.

\section{Measurements}

Parasternal Intercostal Electromyography. Self-adhesive silver-silver chloride electrodes (Kendall Arbo, Tyco Healthcare, Neustadt, Germany) were placed directly adjacent to the sternal border in the second intercostal space bilaterally, with the positive electrode on the right. A reference electrode was placed on the acromion process of the right scapula or the right olecranon in the case of smaller infants. Prior to electrode placement the skin was prepared using an abrasive gel (Nuprep, Weaver and Company, Aurora, CO) and cleaned with an alcohol swab. The EMGpara signals were amplified and bandpass filtered between 10 and 2,000 Hz, (1,902 Biomedical Amplifier, Cambridge Electronic Design, Cambridge, UK).

EMG signals were acquired (Powerlab 16SP, ADInstruments, Sydney, Australia), and displayed on a laptop computer running LabChart software (Version 7.2, ADInstruments Pty, Colorado Springs, CO) with an analogue to digital sampling rate of $4 \mathrm{kHz}$. An adaptive mains filter was applied to the EMG signals by the acquisition hardware to minimize mains frequency interference. A postacquisition digital band-pass filter was applied between 20 and 1,000 Hz to isolate the frequencies of interest. Raw EMG signals were converted to root mean square (RMS) using a moving average window of $50 \mathrm{~ms}$.

Phasic electromyographic activity occurring between QRS complexes was identified and peak RMS EMGpara calculated for each breath in which the peak EMG activity was uncontaminated by ECG activity. Mean peak RMS EMGpara per breath (referred throughout as "EMGpara") was calculated over each stable minute of recording. 
In the PICU patients, only patient-triggered breaths during MV were analyzed. The final stable minute of each subject's recording period was reported.

As surface EMG techniques can be affected by a number of factors related to individual body composition, particularly subcutaneous adipose tissue, the raw EMGpara signal has often been normalized to that obtained during a maximal inspiratory effort $(9,13,14,22)$. This method limits the application of the technique to individuals able to reliably comply with such maneuvers, but may also result in greater risk of inaccuracy due to the risk of submaximal efforts or contamination of the EMGpara signal through recruitment of accessory muscle groups even in those subjects deemed capable of performing such maneuvers. The utility of the raw (nonnormalized) EMGpara signal has not, to date, been explored. In this study, we therefore investigated the utility of both the raw EMGpara signal (in microvolts) and, where feasible, the normalized value (EMGpara expressed as a percentage of maximum, EMGpara\%max).

Other Measurements. Respiratory flow was measured in the PICU patients only, using a pneumotachograph (4,500 series, Hans Rudolph, Kansas City, MO) inserted between the ETT connector and the ventilator manifold, and associated differential pressure transducer (MP45, Validyne, Northridge, CA) and carrier amplifier (CD280, Validyne, Northridge, CA). Flow was acquired simultaneously with EMGpara (Powerlab 16SP), with an analogue to digital sampling rate of $100 \mathrm{~Hz}$. $V_{\mathrm{T}}$ was derived as the digital integral of respiratory flow. Measures of respiratory duty cycle (ratio of inspiratory time to total breath time (Ti:Ttot)) and RR were also derived from the flow signal. The RSBI was also calculated by dividing $V_{\mathrm{T}}$ corrected for body weight by RR (29). The ratio of $V_{\mathrm{T}}$ corrected for body weight $(\mathrm{ml} / \mathrm{kg}$ ) to EMGpara was also used to provide an index of NVE. As with EMGpara, values from the final stable minute of the recording period were reported.

\section{Protocol}

Protocol-Healthy and Wheezy Children. Children were seated comfortably in a chair with trunk and legs supported and arms resting on thighs. EMGpara was recorded during 10 min of steady tidal breathing at rest. Portions of the recording during which the child was talking, laughing, moving their upper limbs or not fully supported in the chair were annotated and subsequently excluded from analysis. At the end of this period, the child was encouraged to perform maximal inspirations to total lung capacity in order to elicit maximal EMGpara activity. Verbal encouragement was provided to promote maximal effort from the subject while avoiding upper limb and trunk movement. The intensity of the effort exerted by the patient was assessed by the investigator, as was the quality of the EMGpara trace during the maneuver. Three efforts with peak EMGpara activity within $10 \%$ of one another were required. If the visual appearance of the EMGpara trace suggested contamination from other muscle groups, the effort was deemed technically unacceptable. The highest peak EMGpara value measured during these maneuvers was reported as the maximum EMGpara activity (maxEMGpara).

In a subset of healthy children, repeat measurements of EMGpara were obtained within the same testing session to assess repeatability of the measure. Electrodes were removed following the initial measurement and then skin prepared and electrodes reapplied at least $1 \mathrm{~h}$ later, with measurement of EMGpara performed as described above.

Protocol-PICU Patients. Patients were positioned supine during measurements and all had stable heart rate and blood pressure. ETT suction was performed $10-15 \mathrm{~min}$ prior to study measurements being undertaken. EMGpara, airway pressure, and respiratory flow were recorded during $10 \mathrm{~min}$ of supported $\mathrm{MV}$ on the settings determined by the clinical team. All patients were ventilated (Evita XL ventilator, Dräger, Lübeck, Germany) on spontaneous modes, CPAP plus pressure support or bilevel positive airways pressure. Children were then switched to $5 \mathrm{cmH}_{2} \mathrm{O}$ CPAP for $10 \mathrm{~min}$, during which EMGpara, airway pressure, and flow were measured continuously. $\mathrm{FiO}_{2}$ was kept constant throughout. All ventilator settings and standard clinical variables were recorded. Recordings were annotated to ensure any periods incorporating coughing or movement were excluded from analysis.

\section{Statistical Analyses}

The Wilcoxon matched pairs test was used to compare withinsubject changes between conditions in the preschool wheeze and PICU groups. Spearman's correlation coefficients were calculated to assess relationships between variables. Logarithmic transformation was applied to the healthy data due to the nonnormal distribution of both the data and residuals. Linear regression analysis was performed to determine a prediction equation for normal logEMGpara based on the healthy control subjects' values, with the Wald-Wolfowitz runs test applied to assess for deviation from linearity. Predicted logEMGpara was derived from the equation

\section{Predicted $\operatorname{logEMGpara}=-0.02347 \times$ age +0.9332}

EMGpara data from patient groups were log-transformed and then expressed as residual standard deviation from the predicted mean logEMGpara value ("z-score"), thereby accounting for differing variability in EMGpara across the age range studied. Comparisons between healthy children and the two conditions for each patient group were made using the Kruskal-Wallis test with Dunn's correction for multiple comparisons applied.

Additional exploratory analyses were performed to investigate potential clinical applications of the EMGpara technique. A threshold for a statistically significant reduction in EMGpara following bronchodilator was determined in the preschool wheeze group from the mean interminute coefficient of variation of the EMGpara signal multiplied by 1.65 (30). ROC analysis was used in the PICU cohort to evaluate the predictive power of measured variables with respect to extubation outcome, using thresholds demonstrating the optimum balance between sensitivity and specificity.

\section{Sample Size Calculation}

Due to the exploratory, observational, feasibility nature of the work, no a priori sample size calculation was undertaken for this study. The three study groups represented convenience samples.

\section{ACKNOWLEDGMENTS}

This research was supported by the National Institute for Health Research (NIHR) Biomedical Research Centre at Guy's and St Thomas' NHS Foundation Trust and King's College London. The views expressed are those of the author(s) and not necessarily those of the NHS, the NIHR, or the Department of Health. A.G. is an NIHR senior investigator.

\section{STATEMENT OF FINANCIAL SUPPORT}

Guy's and St Thomas' Charity (grant no. R070709), London, UK; Asthma UK (grant no. 10/019), London, UK; King's Health Partners Challenge Fund (grant no. R120520), London, UK; and the Rosetrees Trust (grant no. CM128), London, UK.

Disclosure: There is no conflict of interest to declare.

\section{REFERENCES}

1. Eber E, Midulla F. ERS Handbook: Paediatric Respiratory Medicine. Sheffield: European Respiratory Society, 2013: 33-43.

2. Newth CJ, Venkataraman S, Willson DF, et al.; Eunice Shriver Kennedy National Institute of Child Health and Human Development Collaborative Pediatric Critical Care Research Network. Weaning and extubation readiness in pediatric patients. Pediatr Crit Care Med 2009;10:1-11.

3. British Thoracic Society, Scottish Intercollegiate Guidelines Network. British Guideline on the Management of Asthma: A National Clinical Guideline, 2014. https://www.brit-thoracic.org.uk/document-library/ clinical-information/asthma/btssign-asthma-guideline-2014. Accessed 19 November 2015.

4. Rosenfeld M, Allen J, Arets BH, et al.; American Thoracic Society Assembly on Pediatrics Working Group on Infant and Preschool Lung Function Testing. An official American Thoracic Society workshop report: optimal lung function tests for monitoring cystic fibrosis, bronchopulmonary 


\section{Articles | MacBean etal.}

dysplasia, and recurrent wheezing in children less than 6 years of age. Ann Am Thorac Soc 2013;10:S1-S11.

5. Mioxham J, Jolley C. Breathlessness, fatigue and the respiratory muscles. Clin Med (Lond) 2009;9:448-52.

6. Jolley CJ, Luo YM, Steier J, et al. Neural respiratory drive in healthy subjects and in COPD. Eur Respir J 2009;33:289-97.

7. De Troyer A, Estenne M. Coordination between rib cage muscles and diaphragm during quiet breathing in humans. J Appl Physiol 1984;57:899-906.

8. Reilly CC, Jolley CJ, Ward K, MacBean V, Moxham J, Rafferty GF. Neural respiratory drive measured during inspiratory threshold loading and acute hypercapnia in healthy individuals. Exp Physiol 2013;98:1190-8.

9. Reilly CC, Ward K, Jolley CJ, et al. Neural respiratory drive, pulmonary mechanics and breathlessness in patients with cystic fibrosis. Thorax 2011;66:240-6.

10. MacBean V, Sutton TG, Pringle CL, et al. Extubation outcome and parasternal intercostal electromyography in children. Am J Respir Crit Care Med 2014;185:A6145.

11. MacBean V, Siddiqui MAF, Greenough A, Moxham J, Rafferty GF. Neural respiratory drive in healthy children. Am J Respir Crit Care Med 2012;185:A6145.

12. MacBean V, Greenough A, Moxham J, Rafferty GF. Parasternal intercostal electromyography in the assessment of preschool wheeze. Eur Respir J 2013;42:4980s.

13. Steier J, Jolley CJ, Polkey MI, Moxham J. Nocturnal asthma monitoring by chest wall electromyography. Thorax 2011;66:609-14.

14. Murphy PB, Kumar A, Reilly C, et al. Neural respiratory drive as a physiological biomarker to monitor change during acute exacerbations of COPD. Thorax 2011;66:602-8.

15. Harikumar G, Moxham J, Greenough A, Rafferty GF. Measurement of maximal inspiratory pressure in ventilated children. Pediatr Pulmonol 2008;43:1085-91.

16. De Troyer A, Sampson MG. Activation of the parasternal intercostals during breathing efforts in human subjects. J Appl Physiol 1982;52:524-9.

17. Maarsingh EJ, van Eykern LA, Sprikkelman AB, Hoekstra MO, van Aalderen WM. Respiratory muscle activity measured with a noninvasive EMG technique: technical aspects and reproducibility. J Appl Physiol (1985) 2000;88:1955-61.
18. Maarsingh EJ, van Eykern LA, de Haan RJ, Griffioen RW, Hoekstra MO, van Aalderen WM. Airflow limitation in asthmatic children assessed with a non-invasive EMG technique. Respir Physiol Neurobiol 2002;133: 89-97.

19. Gaultier C, Perret L, Boule M, Buvry A, Girard F. Occlusion pressure and breathing pattern in healthy children. Respir Physiol 1981;46:71-80.

20. Saboisky JP, Gorman RB, De Troyer A, Gandevia SC, Butler JE. Differential activation among five human inspiratory motoneuron pools during tidal breathing. J Appl Physiol (1985) 2007;102:772-80.

21. Reilly CC, Jolley CJ, Elston C, Moxham J, Rafferty GF. Measurement of parasternal intercostal electromyogram during an infective exacerbation in patients with cystic fibrosis. Eur Respir J 2012;40:977-81.

22. Steier J, Jolley CJ, Seymour J, Roughton M, Polkey MI, Moxham J. Neural respiratory drive in obesity. Thorax 2009;64:719-25.

23. Maarsingh EJ, Oud M, van Eykern LA, Hoekstra MO, van Aalderen WM. Electromyographic monitoring of respiratory muscle activity in dyspneic infants and toddlers. Respir Physiol Neurobiol 2006;150:191-9.

24. Maarsingh EJ, van Eykern LA, Sprikkelman AB, van Aalderen WM. Histamine induced airway response in pre-school children assessed by a non-invasive EMG technique. Respir Med 2004;98:363-72.

25. Roussos C, Fixley M, Gross D, Macklem PT. Fatigue of inspiratory muscles and their synergic behavior. J Appl Physiol Respir Environ Exerc Physiol 1979;46:897-904.

26. Castro-Rodriguez JA, Rodriguez-Martinez CE, Custovic A. Infantile and preschool asthma. European Respiratory Monograph 2012;56:10-21.

27. Nielsen KG, Bisgaard H. Discriminative capacity of bronchodilator response measured with three different lung function techniques in asthmatic and healthy children aged 2 to 5 years. Am J Respir Crit Care Med 2001;164:554-9.

28. Harikumar G, Egberongbe Y, Nadel S, et al. Tension-time index as a predictor of extubation outcome in ventilated children. Am J Respir Crit Care Med 2009;180:982-8.

29. Baumeister BL, el-Khatib M, Smith PG, Blumer JL. Evaluation of predictors of weaning from mechanical ventilation in pediatric patients. Pediatr Pulmonol 1997;24:344-52.

30. Pennock BE, Rogers RM, McCaffree DR. Changes in measured spirometric indices. What is significant? Chest 1981;80:97-9. 